\title{
Stability analysis in alpha helical protein model with higher order molecular excitations
}

\author{
S. Saravana Veni \\ Department of Physics, Ponjesly College of Engineering, Nagercoil- 629001
}

The dynamics of continuum alpha helical proteins with dipole and quadrupole type molecular excitations is presented by deriving Hamilton's equations of motion. The condition for modulation instability and formation of spatial solitons is investigated. The standard modulation instability mechanism has shown which can initiate the process of the formation of localized pulses.

\section{Introduction}

Solitons have been throughly studied in diverse fields of physics such as nonlinear optics, Bose Einstein Condensates, plasma and water waves. An important biological problem is the mechanism of energy transfer and storage in alpha helical proteins. Solitons in alpha helical proteins was made by Davydov in an attempt to solve an outstanding riddle of biochemistry. The analytical theory of this soliton has been discussed in a number of papers by Davydov and his co workers from which it is seen that anharmonicity in the longitudinal hydrogen bond plays a major role in effecting the mechanisms. Due to its transparency and seminal properties, Davydov's model continues to encourage intense work regarding the research of the nonlinear treatment of molecular systems. In this paper, the dynamics of alpha helical proteins with dipole and quadrupole type molecular excitations is considered. The energy transfer in alpha helical proteins is studied by the production of localized pulses due to modulation instability (MI). The scheme of MI analysis is presented by deriving the generalized dispersion which relates the frequency and wavenumber of modulating perturbations and the possible consequences for the energy localization for the higher order alpha helical proteins is investigated. The basic results which are given display the MI gain as a function of frequency of infinitesimal modulation peturbations. Typical outcomes of nonlinear development of the MI, in the form of regular and irregular patterns are plotted.

\section{The Model}

The model is to be considered representing the peptide group of the alpha helical protein chain along the hydrogen bonding spine with dipole and quadrupole type molecular excitations and nearest and next nearest neighbour molecular interactions of the form $[1,2]$,

$$
\begin{aligned}
H= & \sum_{n}\left\{B _ { n } ^ { \dagger } \left[E_{0} B_{n}+E_{1} B_{n} B_{n}^{\dagger} B_{n}-J_{0}\left(B_{n+1}+\lambda B_{n-1}\right)-J_{1}\left(B_{n+1} B_{n}^{\dagger} B_{n+1}\right.\right.\right. \\
& \left.+\lambda B_{n-1} B_{n}^{\dagger} B_{n-1}\right)+\frac{1}{2}\left(\frac{p_{n, \alpha}^{\mathrm{n}}}{m}+K_{1}\left(u_{n, \alpha}-u_{n-1, \alpha}\right)^{2}+K_{2}\left(u_{n}\right.\right. \\
& \left.\left.-u_{n-2}\right)^{2}\right)+\chi_{1}\left(u_{n+1}-u_{n-1}\right) B_{n}^{\dagger} B_{n}+\chi_{2}\left(u_{n+2}-u_{n}\right) B_{n}^{\dagger} B_{n+1} \\
& \left.\left.+\chi_{3}\left(u_{n}-u_{n-2}\right) B_{n}^{\dagger} B_{n-1}+\chi_{4}\left(u_{n+1}-u_{n-1}\right) B_{n}^{\dagger} B_{n} B_{n}^{\dagger} B_{n}\right]\right\}
\end{aligned}
$$

where the summation with respect to $n$ runs over the unit cells $(H-N-C=O)$ along the infinite hydrogen bonding spines. $B_{n}^{\dagger}$ and $B_{n}$ represent the creation and annihilation operators for the internal molecular excitations of the peptide group labelled by $n$.

In order to understand the underlying dynamics using the wave function for collective excitations of the chain the equations of motion is derived which can be written as

$$
\begin{aligned}
& i \hbar \frac{d a_{n}}{d t}=\left(E_{0}+W\right) a_{n}+2 E_{1} a_{n}^{*} a_{n}^{2}-J_{0}\left(a_{n+1}+\lambda a_{n-1}\right)-2 J_{1}\left(a_{n+1}^{2}\right. \\
& \left.\quad+\lambda a_{n-1}^{2}\right) a_{n}^{*}+\chi_{1}\left(b_{n+1}-b_{n-1}\right) a_{n}+\chi_{2}\left(b_{n+2}-b_{n}\right) a_{n+1} \\
& +\chi_{3}\left(b_{n}-b_{n-2}\right) a_{n-1}+2 \chi_{4}\left(b_{n+1}-b_{n-1}\right) a_{n}^{2} a_{n}^{*}
\end{aligned}
$$




$$
\begin{aligned}
& m \frac{d^{2} b_{n}}{d t^{2}}=K_{1}\left(b_{n+1}+b_{n-1}-2 b_{n}\right)+K_{2}\left(b_{n+2}+b_{n-2}-2 b_{n}\right)+\chi_{1}\left(\left|a_{n+1}\right|^{2}\right. \\
& \left.\quad-\left|a_{n-1}\right|^{2}\right)+\chi_{2}\left(a_{n-2}^{*} a_{n-1}-a_{n}^{*} a_{n+1}\right)+\chi_{3}\left(a_{n+2}^{*} a_{n+1}-a_{n}^{*} a_{n-1}\right) \\
& \quad+\chi_{4}\left(\left|a_{n+1}\right|^{4}-\left|a_{n-1}\right|^{4}\right) .
\end{aligned}
$$

where,

$$
W=\frac{1}{2}\left(\frac{\phi_{n, \alpha}^{x}}{m}+K_{1}\left(b_{n, \alpha}-b_{n-1, \alpha}\right)^{2}+K_{2}\left(b_{n, \alpha}-b_{n-2, \alpha}\right)^{2}\right)
$$

is the deformation energy of the spines.

After making the continuum approximation followed by some simplifications equations (2) become the completely integrable fourth order NLS equation

$$
\begin{aligned}
& i q_{t}+\frac{1}{2} q_{\xi \xi}+|q|^{2} q+\gamma\left\{q_{\xi \xi \xi \xi}+6 q^{*} q_{\xi}^{2}+4 q\left|q_{\xi}\right|^{2}+8|q|^{2} q_{\xi \xi}\right. \\
& \left.+2 q^{2} q_{\xi \xi}^{*}+6|q|^{4} q\right\}=0 .
\end{aligned}
$$

The equation (4) is the completely integrable higher order NLS equation which represent the dynamics of higher order alpha helical proteins with nearest and next nearest neighbour interactions [1]. The integrablity and multisoliton solutions has been studied very recently [2].

\section{Stability of soliton in higher order continuum alpha helical proteins}

As is well known, the nonlinear physical systems may exhibit an instability that leads to a self induced modulation of the steady state as a result of an interplay between nonlinear and dispersive effects. As has been pointed out, modulation instability is responsible for energy localization and formation of localized pulses. Modulation Instability is one of the most fundamental effects associated with wave propagation in nonlinear media. It signifies the exponential growth of a small perturbation of the amplitude of the wave during propagation. The presence of modulation instability is closely connected with the existence of soliton solutions to the nonlinear systems $[3,4]$.

An equilibrium state can be obtained by inserting the assumption

$$
q=q_{0} e^{i \omega t}
$$

where $\omega$ is the frequency and $q_{0}$ is the amplitude. Substituting equation (5) into the equation (4) we get the amplitude dependent relationship,

$$
\omega=\left|q_{0}\right|^{2}\left(1+6 \gamma\left|q_{0}\right|^{2}\right),
$$

known as the dispersion relation. Now we analyze the linear stability of (5) by considering perturbed solution

$$
q=\left(q_{0}+\varepsilon q_{1}\right) e^{i \omega t}
$$

We substitute the perturbed solution (7) into the equation (4) and the first order terms of $\varepsilon$ yields the linearized equation for the perturbation of the form

$$
\begin{aligned}
& -\omega q_{1}+i q_{1, t}+\frac{1}{2} q_{1, x x}+q_{0}^{2} q_{1}^{*}+2\left|q_{0}\right|^{2} q_{1}+\gamma\left(q_{1, x x x x}+8\left|q_{0}\right|^{2} q_{1, x x}\right. \\
& +2 q_{0}^{2} q_{1, x x}^{*}+12 q_{0}^{3} q_{0}^{*} q_{1}^{*}+18 q_{0}^{2}\left(q_{0}^{*}\right)^{2} q_{1}=0 .
\end{aligned}
$$

Writing, $q_{1}=u+i v$ and separating the real and imaginary parts of the linearized equation (8), we get

$$
\begin{aligned}
& -\omega u-v_{t}+\frac{1}{2} u_{x x}+q_{0}^{2} u+2\left|q_{0}\right|^{2} u+\gamma\left(u_{x x x x}+8\left|q_{0}\right|^{2} u_{x x}+2 q_{0}^{2} u_{x x}\right. \\
& \left.+12 q_{0}^{3} q_{0}^{*} u+18 q_{0}^{2}\left(q_{0}^{*}\right)^{2} u\right)=0, \\
& -\omega v+u_{t}+\frac{1}{2} v_{x x}-q_{0}^{2} v+2\left|q_{0}\right|^{2} v+\gamma\left(v_{x x x x}+8\left|q_{0}\right|^{2} v_{x x}-2 q_{0}^{2} v_{x x}\right. \\
& \left.-12 q_{0}^{3} q_{0}^{*} v+18 q_{0}^{2}\left(q_{0}^{*}\right)^{2} v\right)=0 .
\end{aligned}
$$

By introducing the transformations,

$$
\begin{aligned}
& u=u_{0} e^{i(K x-\Omega t)}, \\
& v=v_{0} e^{i(K x-\Omega t)},
\end{aligned}
$$

where $K$ and $\Omega$ are the perturbation wave number and the frequency respectively and after some straightforward calculations, we obtain the nonlinear dispersion relation

$$
\Omega^{2}=\alpha_{1} K^{8}+\alpha_{2} K^{6}+\alpha_{3} K^{4}+\alpha_{4} K^{2}+\alpha_{5}
$$

National Conference on Current Advancements in Physics $3^{\text {rd }} \& 4^{\text {th }}$ February 2017

Department of Physics, St. John's College, Palayamkottai-627 002, Tamilnadu, India. DOI 10.9790/4861-17002011215 
where

$$
\begin{aligned}
& \alpha_{1}=\gamma^{2}, \\
& \alpha_{2}=-\gamma\left(1+16 \gamma\left|q_{0}\right|^{2}\right), \\
& \alpha_{3}=\frac{1}{4}+10\left|q_{0}\right|^{2} \gamma+\left(88\left(q_{0}^{*}\right)^{2}+4 q_{0}^{2}\right) q_{0}^{2} \gamma^{2}, \\
& \left.\alpha_{4}=-\left(\left|q_{0}\right|^{2}+\left(28\left|q_{0}\right|^{4}-4 q_{0}^{4}\right) \gamma+\left(192\left|q_{0}\right|^{2}\right)^{3}-48\left|q_{0}\right|^{2} q_{0}^{4}\right) \gamma^{2}\right), \\
& \alpha_{5}=-\left(q_{0}^{4}+24\left(-\left|q_{0}\right|^{6}+\left|q_{0}\right|^{2} q_{0}^{4}\right) \gamma+144\left(-\left|q_{0}\right|^{8}+\left|q_{0}\right|^{2} q_{0}^{2}\right) \gamma^{2}-\left|q_{0}\right|^{2} .\right.
\end{aligned}
$$

Equation (12) gives the dispersion relation which determines $\mathrm{K}$ as a function of $\Omega$ including the MI gain $\sigma=\sqrt{-\Omega^{2}}$. The full spectrum of MI gain is found from the solution of dispersion relation (12).

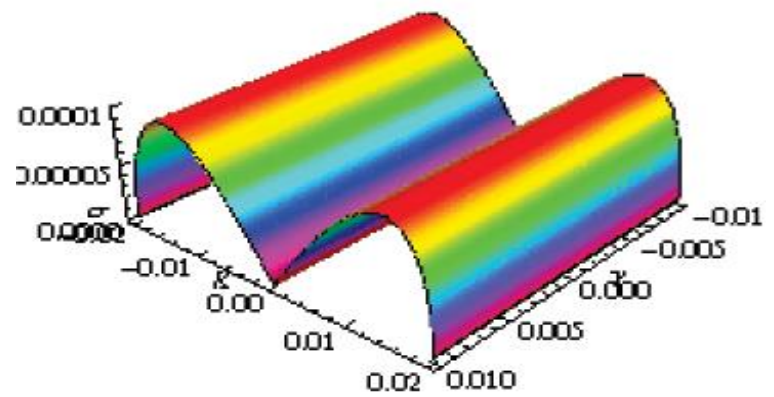

(a)

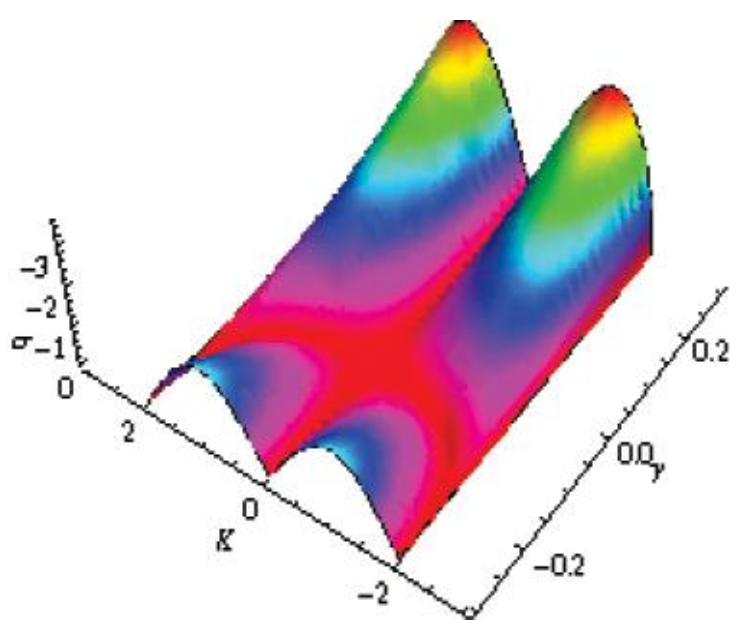

(b)

Figure 1. Modulation instability gain $\sigma=\sqrt{-\Omega^{2}}$ versus perturbation frequency $K$ and the parameter $\gamma$ with the amplitude $q_{0}=0.01$ and $q_{0}=1$.
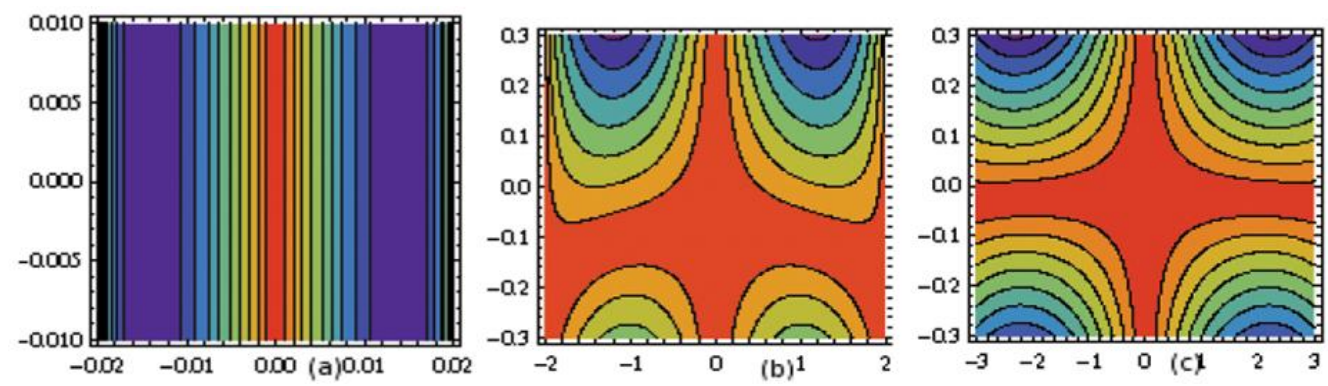

Figure 2. Contour Plots for the modulation instability region with the creation of localised pulses and the soliton interaction in higher order alpha helical proteins for (a) $q_{0}=0.01$, (b) $q_{0}=1$ and (c) $q_{0}=2$. 
In figures (1a) and (1b) the corresponding gain is shown through surface plots against the wavenumber and the frequency of perturbation $\Omega$. From the figure it is observed that in both cases the gain actually depend on any parameters. It is also noteworthy that the gain curves are quite smooth for the amplitude $\mathrm{q}_{0}<1$, but not for $\mathrm{q}_{0} \geq 1$. The corresponding contour plots are given in figure 2 . According to the figure the instability is predicted for a wave with wavenumbers. Therefore by using realistic values of parameters, the investigation is carried out the bearing of localized structures in the molecular chain [5]. This also confirms the fact that MI is a way among others through which energy could be transported in nonlinear lattices. That is, because as already stated, MI is the way through which soliton-like structures emerge in nonlinear systems. Physically, modulation stability means that small amplitude waves can propagate along with the background intense soliton, although their propagation parameter $\Omega$ depends on the soliton intensity $q_{0}^{2}$.

\section{Conclusion}

The aim of this paper is to study the energy transfer in higher order alpha helical proteins. For this the model representing the dynamics of single hydrogen bonding molecular chain including dipole and quadrupole type molecular excitations and interactions between nearest and next nearest neighbours is considered. The scheme of modulation instability analysis is investigated. The linear stability analysis allows to identify the particular regions in the parameter space where MI may occur and estimates the growth rate of perturbation. Typical dependence of the MI gain on the perturbation frequency were obtained.

\section{References}

[1]. M. Daniel, M. M. Latha, Physica A 240 (1997) 526.

[2]. S. Saravana Veni, M. M. Latha, Physica A 407 (2014) 76.

[3]. K. Porsezian, R. Murali, Boris A. Malomed and R. Ganapathy, Chaos, Solitons and Fractals 40 (2009) 1907.

[4]. H. P. E. Fouda, C. B. Tabi, A. Mohamadou and T. C. Kofane, J. Phys. Condens. Matter 23 (2011) 375104.

[5]. S. Saravana Veni, M. M. Latha, PhysicaA 396 (2014) 29. 